\title{
CONSTRUCTION OF THE NEW HIGH-PRECISION MOON ROTATION SERIES AT A LONG TIME INTERVALS
}

\author{
V.V. Pashkevich, G.I. Eroshkin \\ Central (Pulkovo) Astronomical Observatory of RAS \\ Pulkovskoe shosse, 65/1, 196140, St.Petersburg, Russia \\ e-mails: pashvladvit@yandex.ru , eroshkin@gao.spb.ru
}

\begin{abstract}
The main purposes of this research are the construction of the new highprecision Moon Rotation Series (MRS2011), dynamically adequate to the DE404/LE404 and the DE406/LE406 ephemeris, over long time intervals. The comparison of the new highprecision Moon Rotation solutions of MRS2011 with the solution of MRS2010 (Pashkevich and Eroshkin, 2010), which is dynamically adequate to the DE200/LE200 ephemeris over 418.9 year time interval, is performed. The dynamics of the rotational motion of the Moon is studied numerically by using Rodrigues-Hamilton parameters over 418.9, 2000 and 6000 years. The numerical solution of the Moon rotation is implemented with the quadruple precision of the calculations. The results of the numerical solution of the problem are compared with the composite semi-analytical theory of the Moon rotation (SMR) (Pashkevich and Eroshkin, 2010) with respect to the fixed ecliptic of epoch J2000. The initial conditions of the numerical integration are taken from SMR. The investigation of the discrepancies is carried out by the least squares and spectral analysis methods for the Newtonian case. All the secular, periodic and Poisson terms, representing the behavior of the residuals, are interpreted as corrections to SMR semi-analytical theory. As a result, the Moon Rotation Series (MRS2011) is constructed, which is dynamically adequate to the DE404/LE404 and the DE406/LE406 ephemeris over 418.9, 2000 and 6000 years. A numerical solution for the Moon rotation is obtained anew with the new initial conditions calculated by means of MRS2011. The discrepancies between the new numerical solution and the semi-analytical solution of MRS2011 do not surpass 20 mas over 418.9 year time interval, 64 mas over 2000 year time interval and 8 arc seconds over 6000 year time interval. Thus, the result of the comparison demonstrates a good consistency of MRS2011 series with the DE/LE ephemeris.
\end{abstract}

Keywords: the rotation of the Moon, ephemeris, least-squares method, spectral analysis method.

\section{INTRODUCTION}

In the previous research (Pashkevich and Eroshkin, 2010) the high-precision Moon Rotation semi-analytical solutions of MRS2010, dynamically adequate to the DE200/LE200 ephemeris, was constructed over 418.9 year time interval.

The main purposes of this research are the construction of the new high-precision Moon Rotation Series (MRS2011), dynamically adequate to the DE404/LE404 and the 
DE406/LE406 ephemeris, over long time intervals. The mathematical model of the present investigation is identical to that used by Pashkevich and Eroshkin (2010).

\section{MATHEMATICAL MODEL OF THE PROBLEM}

The numerical solution of the problem is obtained by solving the Lagrange differential equations of the second kind for the Moon rotation with respect to the fixed ecliptic and equinox of epoch $\mathrm{J} 2000$.

The Lagrange differential equations of the second kind

$$
\frac{d}{d t} \frac{\partial L}{\partial \dot{\lambda}_{i}}-\frac{\partial L}{\partial \lambda_{i}}=0, \quad i=0,1,2,3,
$$

are solved, where $L=T+U, T$ is the kinetic energy of the rotational motion of the Moon, $U$ is the force function of the gravitational interaction of the Moon with the disturbing bodies (the Earth, the Sun and the major planets). The force function $U$ is expanded in the spherical harmonics and only the terms with the coefficients $C_{j 0}$ for $j=2,3,4, C_{22}, C_{3 k}, S_{3 k}$ for $\mathrm{k}=1,2,3, \mathrm{C}_{4 \mathrm{k}}, \mathrm{S}_{4 \mathrm{k}}$ for $\mathrm{k}=1, \ldots, 4$ are used. The orbital motions of the disturbing bodies are defined by the DE404/LE404 and the DE406/LE406 ephemeris.

The Rodrigues - Hamilton parameters

$$
\lambda_{0}=\cos \frac{\theta}{2} \cos \frac{\psi+\varphi}{2}, \lambda_{1}=\sin \frac{\theta}{2} \cos \frac{\psi-\varphi}{2}, \lambda_{2}=\sin \frac{\theta}{2} \sin \frac{\psi-\varphi}{2}, \lambda_{3}=\cos \frac{\theta}{2} \sin \frac{\psi+\varphi}{2}
$$

are the functions of the Euler angles $\psi, \theta$ and $\varphi$. These parameters are bounded variables. It is very important for the numerical solution of the problem.

The semi-analytical theory of the Moon rotation (SMR) (Pashkevich and Eroshkin, 2010), which consists of Cassini relations and the semi-analytical solution of the lunar physical libration problem, was constructed with respect to the ecliptic and the equinox of date and then was reduced to the fixed ecliptic of epoch J2000.0.

Since Cassini's relations are not exact, one must consider the perturbed Euler angles:

a) $\varphi+\psi=180^{\circ}+L+\tau$,

b) $\theta=I+\rho$,

c) $\psi=\Omega+\sigma$,

where $\theta$ is the inclination of the lunar equator to the fixed ecliptic J2000, $\psi$ is the longitude of the descending node of epoch J2000 of the lunar equator, $\varphi$ is the proper rotation angle between the descending node of epoch J2000 and the principal axis of the minimum moment of inertia, $I$ is the mean inclination of the lunar equator to the ecliptic of date, $L$ is the mean longitude of the Moon and $\Omega$ is the mean longitude of the ascending node of its orbit; $\tau, \rho$ and $\sigma$ are the perturbing terms of the physical librations in the longitude, in the inclination and in the node longitude, respectively.

The expressions for the classical perturbing terms of the physical librations (Newhall and Williams, 1997) are 


$$
\left.\begin{array}{l}
\Delta \tau=\sum_{j} \sum_{k=0}^{3}\left[\tau_{S j k} \sin \left(v_{j 0}+v_{j 1} t\right)+\tau_{C j k} \cos \left(v_{j 0}+v_{j 1} t\right)\right] t^{k} \\
\Delta \rho=\sum_{j} \sum_{k=0}^{3}\left[\rho_{S j k} \sin \left(v_{j 0}+v_{j 1} t\right)+\rho_{C j k} \cos \left(v_{j 0}+v_{j 1} t\right)\right] t^{k} \\
\Delta I \sigma=I \sum_{j} \sum_{k=0}^{3}\left[\sigma_{S j k} \sin \left(v_{j 0}+v_{j 1} t\right)+\sigma_{C j k} \cos \left(v_{j 0}+v_{j 1} t\right)\right] t^{k}
\end{array}\right\},
$$

where $v_{k 0}, v_{k l}$ are the phases and the frequencies of SMR theory, respectively, $t$ is the time in the Julian days, $\tau_{s j k}, \rho_{s j k}, \sigma_{s j k}, \tau_{c j k}, \rho_{c j k}, \sigma_{c j k}$ are the coefficients of the perturbing terms of the physical librations.

The Moon rotation series SMR consist of 380 periodical terms with the periods from 5.648 days to 84541.30 years. The high-precision numerical integration method (Belikov, 1990), with a number of modifications (Eroshkin et al., 1993), was applied.

\section{ITERATIVE ALGORITHM}

The result of the comparison of the numerical solution and semi-analytical solution SMR is studied by means the iterative algorithm:

1. The numerical solution of the Moon rotation is implemented with the quadruple precision of the calculations. The initial conditions are computed by the semianalytical theory of the Moon rotation (SMR), which corresponds to the fixed ecliptic J2000.0. The discrepancies between the numerical solution and SMR are obtained in Euler angles over all the investigation time intervals with one day spacing.

2. The investigation of the discrepancies is carried out by the least squares method (LSQ) and by the spectral analysis (SA) method (Pashkevich and Eroshkin, 2010). The secular terms are defined by LSQ. The set of the frequencies of SMR theory is used without a change. Only the coefficients of the periodical terms are improved and the coefficients of the Poisson terms are calculated by LSQ and by SA (the spectral analysis scheme presented in Figure 2). The secular, periodic and Poisson terms representing the new high-precision Moon rotation series MRS2011-i (where $i$ is a number of the iteration) are determined.

3. The numerical solution of the Moon rotation is constructed anew with the new initial conditions, which are calculated by MRS2011-i.

4. Steps 2 and 3 (presented in Figure 1) are repeated till the best results for the discrepancies between new numerical solution and new MRS2011-i are obtained. 


\section{Investigation of the discrepancies of the comparison i - iteration}

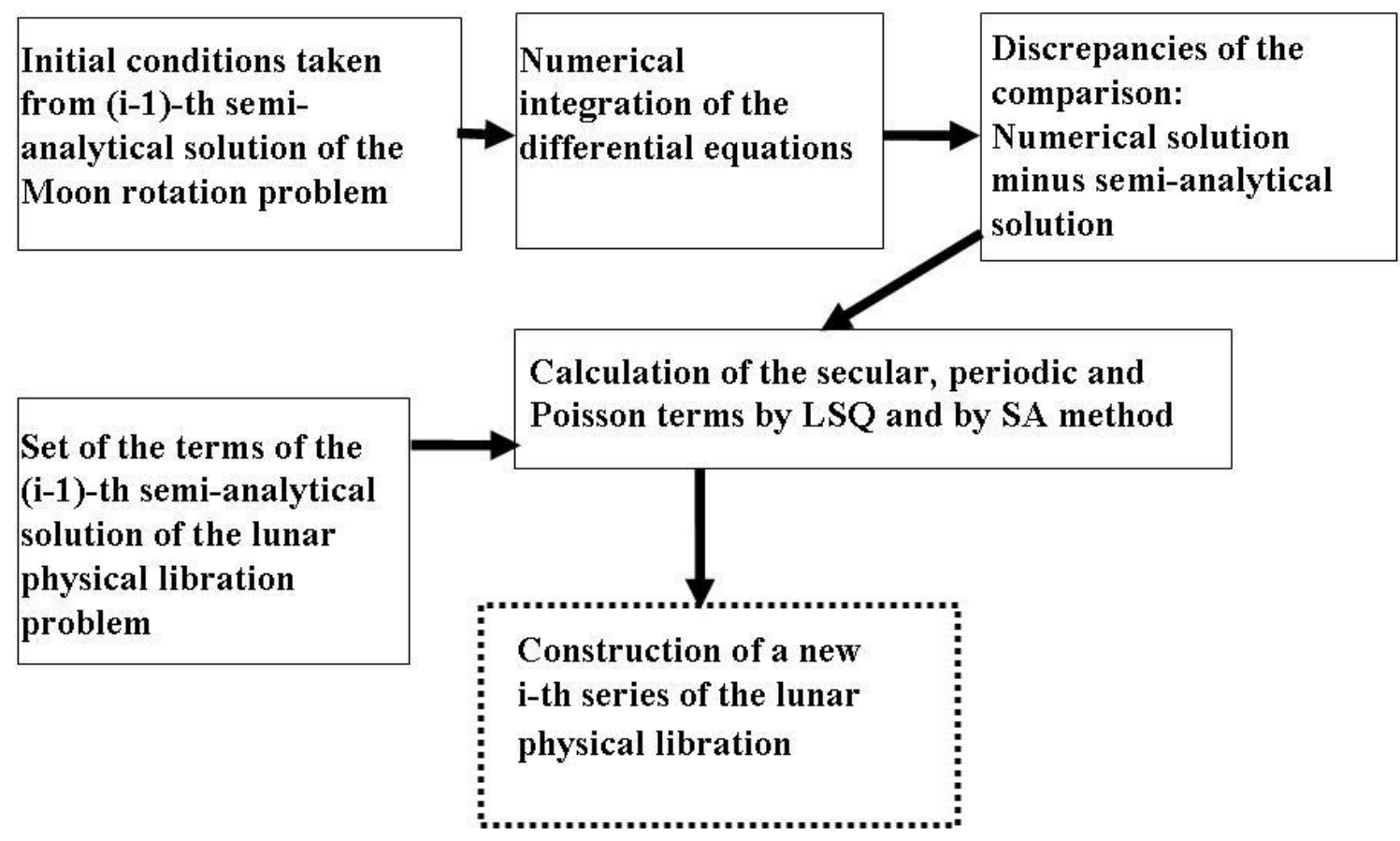

Fig. 1. The iterative algorithm

SA method (presented in Figure 2) is following: The spectrum of the discrepancies between the numerical solution and semi-analytical solution of the (i-1)-th iteration is constructed anew after the removal of every largest residual harmonic from the discrepancies. The set of the frequencies of the semi-analytical solution of the lunar physical libration SMR is used for the power spectrum construction. The amplitudes of the power spectrum are computed by LSQ. Each maximum term of the constructed spectrum is used for the determination of the new coefficients of the periodic and Poisson terms. Every coefficient of the new periodic term equals the sum of the calculated periodic and Poisson terms coefficients of the discrepancies and the coefficients of the corresponding periodic and Poisson terms of the (i1)-th semi-analytical solution of the lunar physical libration problem. The found new harmonic is removed from the discrepancies and from the used set of the frequencies of SMR. This procedure is performed for every harmonic of the set and is accomplished successively up to the least term of the set. The new periodic and Poisson terms representing the new series MRS2011-i are determined. 


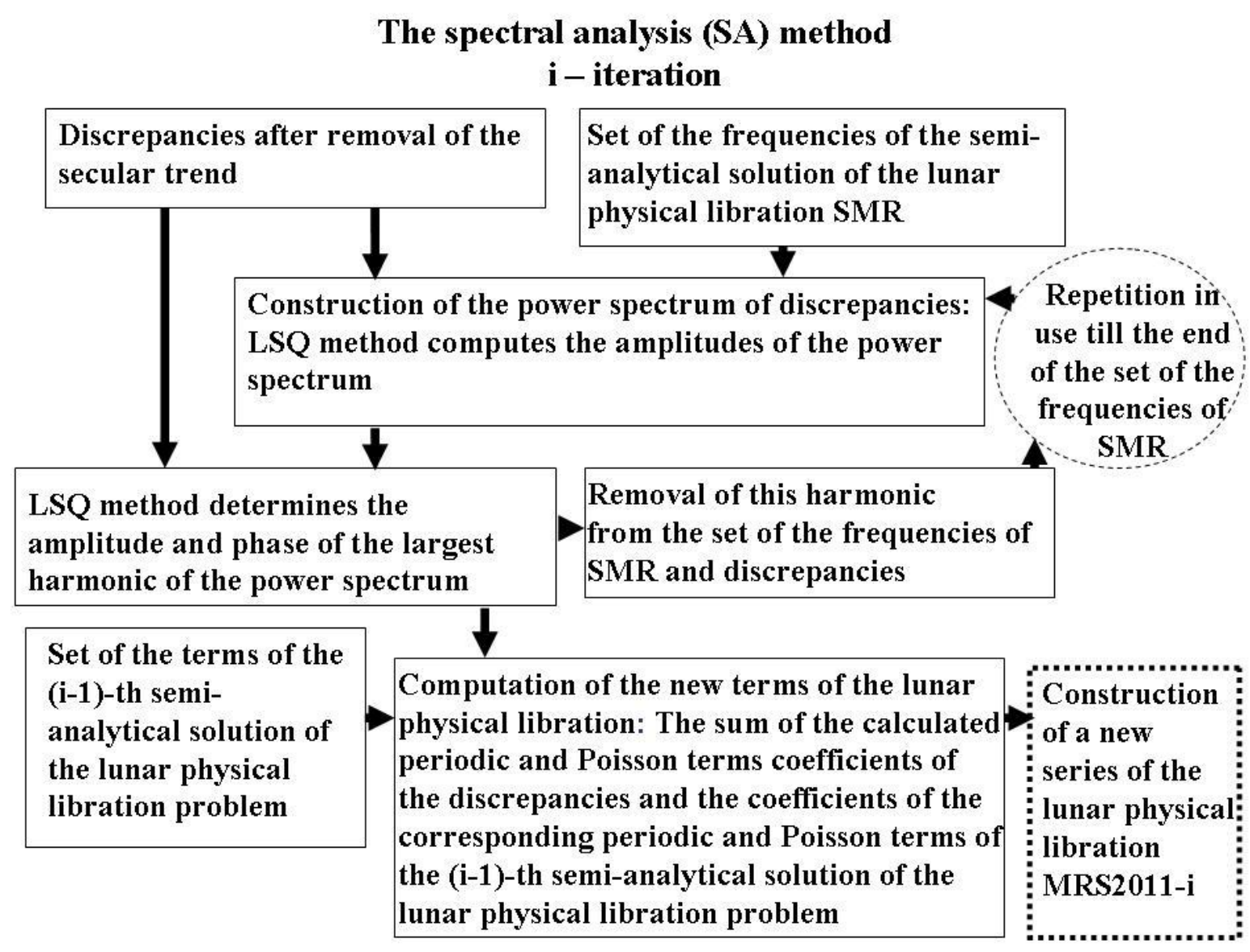

Fig. 2. Spectral analysis scheme

\section{RESULTS}

At first this investigation is carried out on 418.9 years time interval. The discrepancies between the numerical integration of the lunar rotation and the Moon rotation series SMR (for the DE404/LE404 ephemeris (blue color) and for the DE406/LE406 ephemeris (red color)) are obtained in the perturbing terms of the physical librations over 418.9 year time interval with one day spacing (presented in Figure 3). 


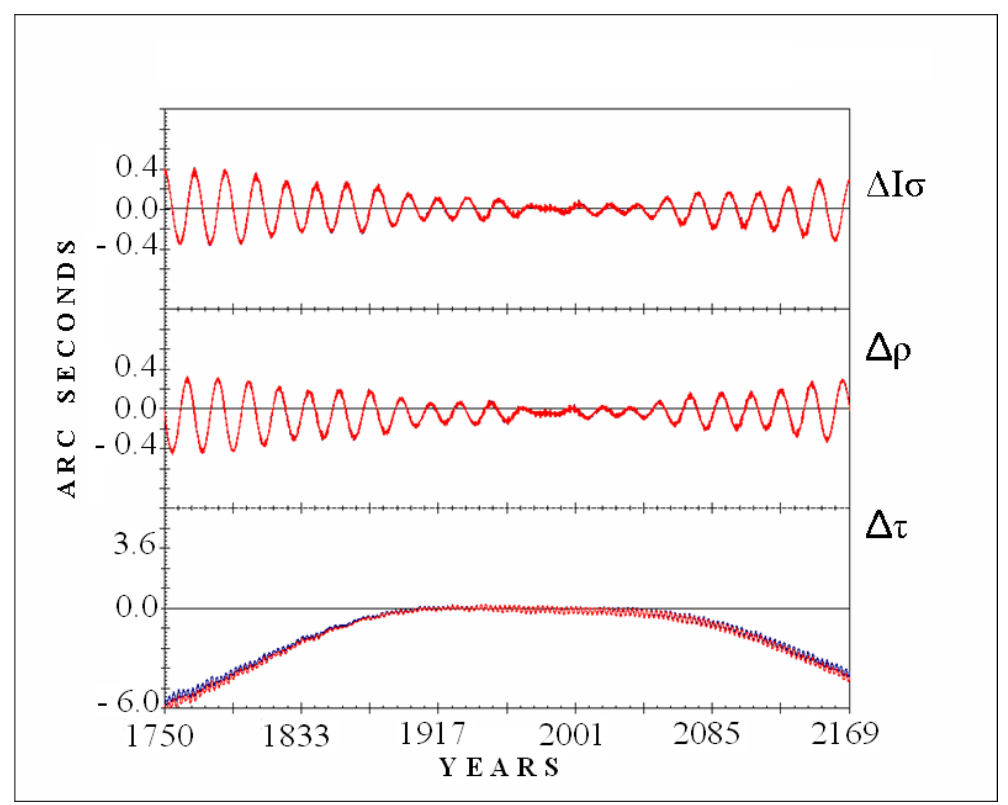

Fig. 3. Discrepancies between the numerical and SMR semi-analytical solutions of the Moon rotation (for the DE404/LE404 ephemeris and for the DE406/LE406 ephemeris) over 418

years

Figure 4 shows that the discrepancy between the new numerical and the new MRS2011A-1 semi-analytical solutions of the Moon rotation for the DE404/LE404 ephemeris (blue color) and the DE406/LE406 ephemeris (red color) are very close to each other after the first iteration of the iterative algorithm.

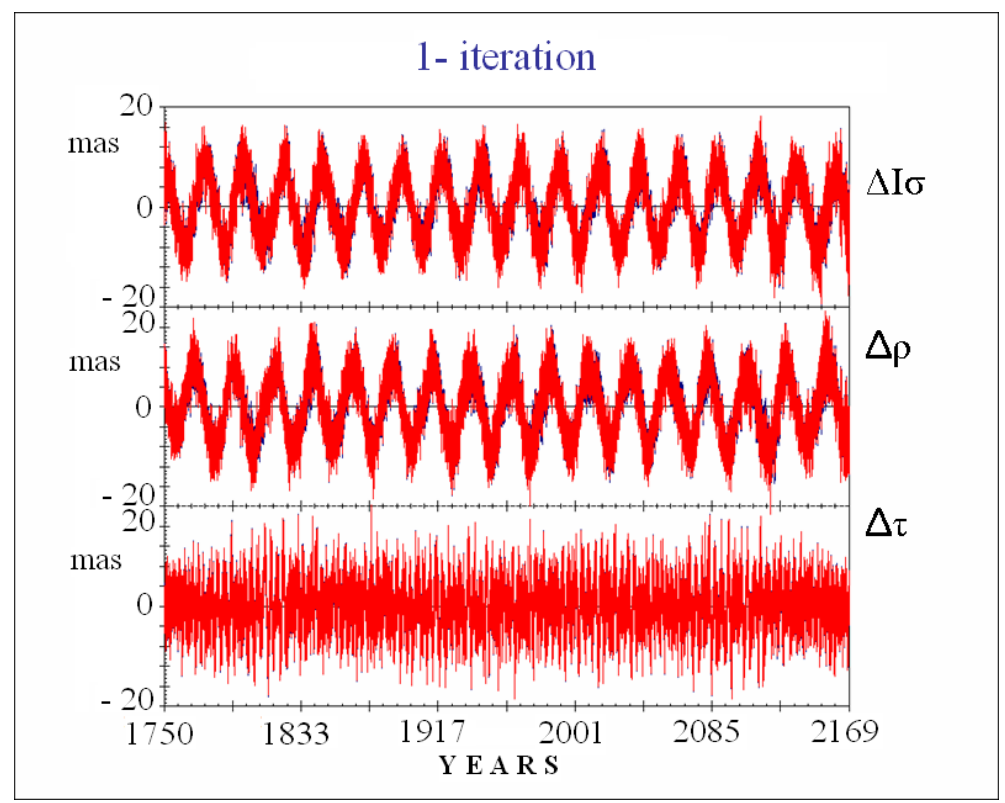

Fig. 4. Discrepancies between the numerical and MRS2011A-1 semi-analytical solutions of the Moon rotation (for the DE404/LE404 ephemeris and for the DE406/LE406 ephemeris)

The residuals between the new numerical and the new MRS2011A-1 semi-analytical solutions of the Moon rotation (for the DE406/LE406 ephemeris - red color), after the first iteration of the iterative algorithm, and the residuals between the numerical and MRS2010 (Pashkevich and Eroshkin, 2010) semi-analytical solutions of the Moon rotation (for the DE200/LE200 ephemeris - blue color) are similar (presented in Figure 5). Namely, the 
periodic and Poisson parts of MRS2011A are very close periodic and Poisson parts of MRS2010, over all time interval of MRS2010, that evidences a good convergency of the iterative algorithm of this investigation.

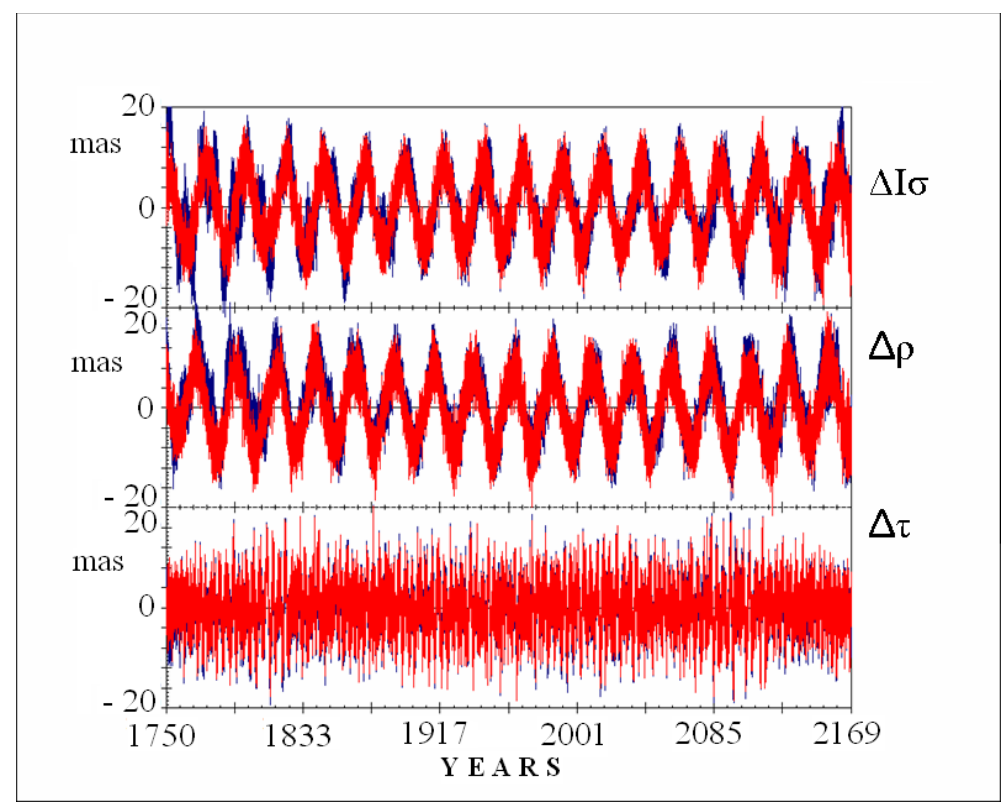

Fig. 5. Discrepancies between the numerical and (MRS2010 (blue color) and MRS2011A-1 (red color)) semi-analytical solutions of the Moon rotation

This investigation is continued on 2000 years time interval. In Figure 6 the discre-pancies are depicted between the numerical and SMR semi-analytical solutions of the Moon rotation (for the DE404/LE404 ephemeris (blue color) and for the DE406/LE406 ephemeris (red color)) over 2000 years.

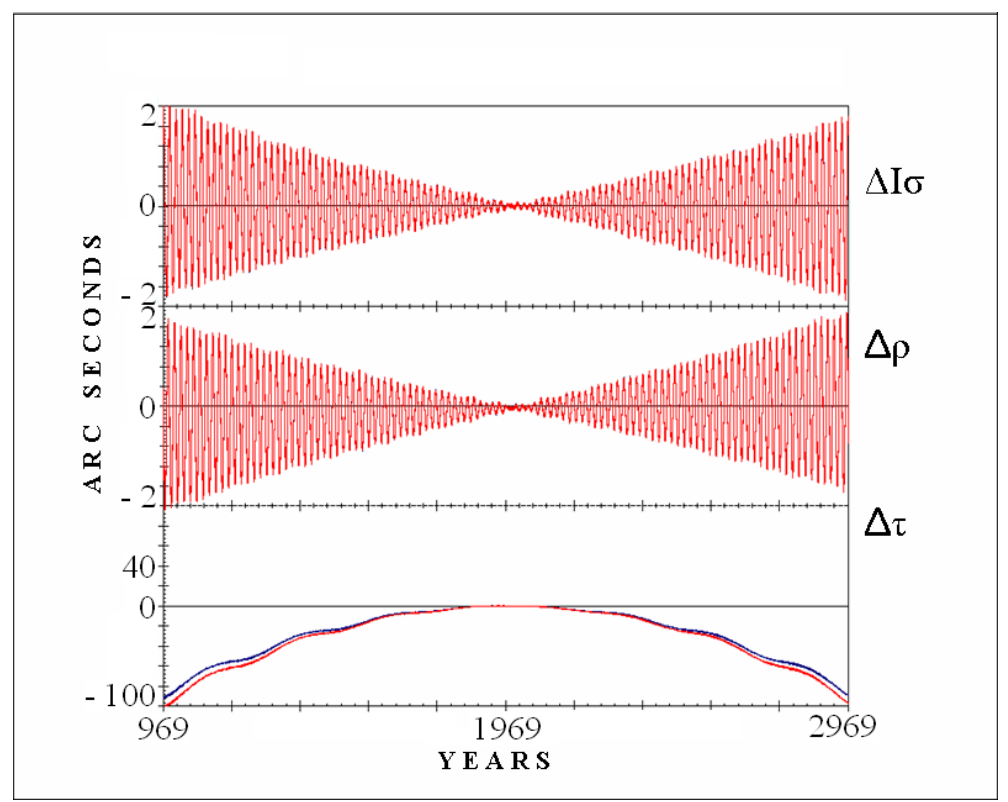

Fig. 6. Discrepancies between the numerical and SMR semi-analytical solutions of the Moon rotation (for the DE404/LE404 ephemeris and for the DE406/LE406 ephemeris) over 2000 
Figure 7 demonstrates that the residuals between the numerical and MRS2011B-1 semianalytical solutions of the Moon rotation for the DE404/LE404 ephemeris (blue color) and the DE406/LE406 ephemeris (red color) are very close to each other after the first iteration of the iterative algorithm. Thus, the second iteration is used only for the investigation with the application of the DE406/LE406 ephemeris.

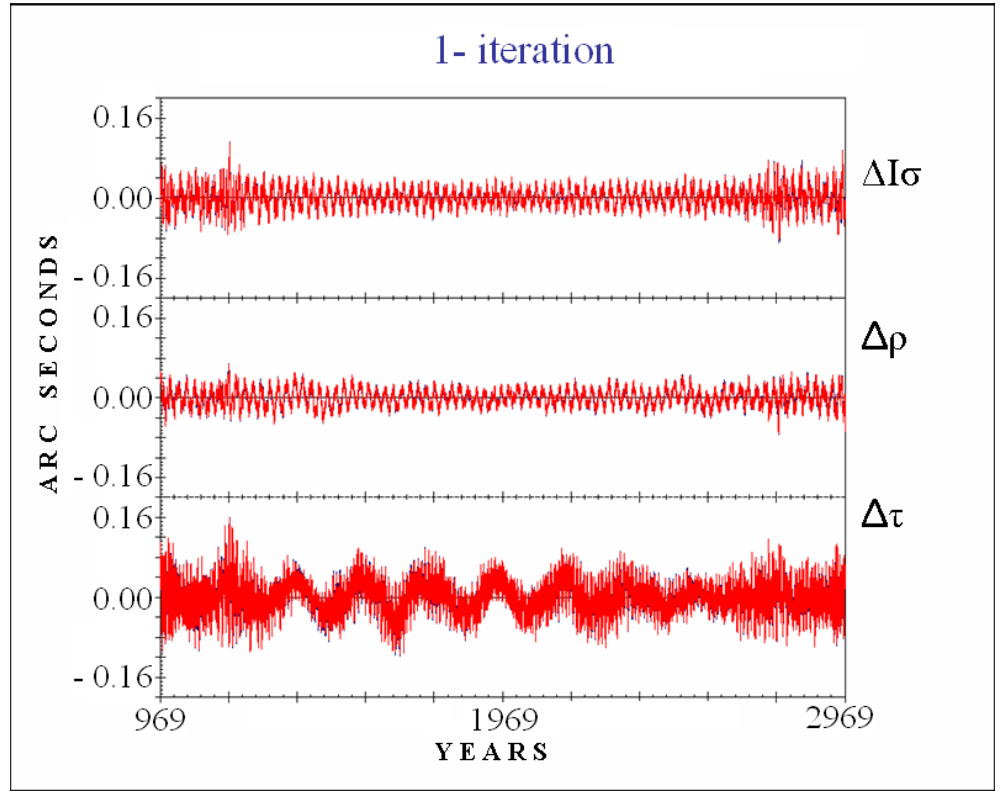

Fig. 7. Discrepancies between the numerical and MRS2011B-1 semi-analytical solutions of the Moon rotation (for the DE404/LE404 ephemeris and for the DE406/LE406 ephemeris)

Figure 8 shows that the residuals between the numerical and MRS2011B-2 (blue color) semianalytical solutions, after the second iteration, and the residuals between the numerical and MRS2011B-3 (red color) semi-analytical solutions, after the third iteration are similar. Then the process of the iterative algorithm is finished at this step.

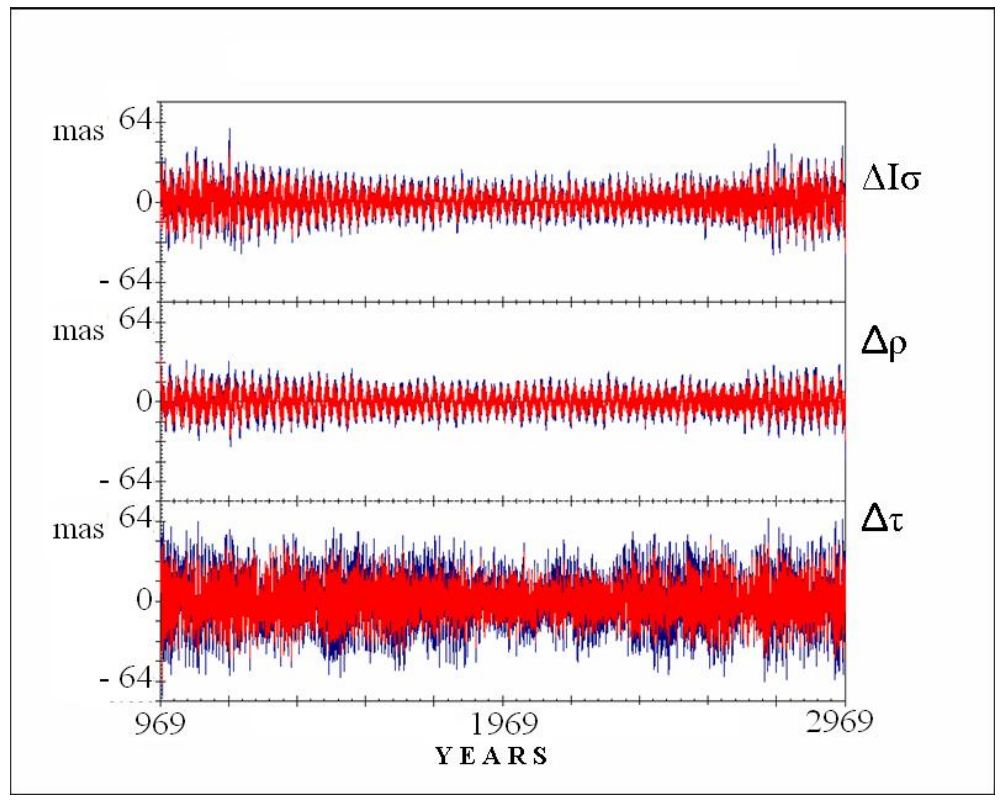

Fig. 8. Discrepancies between the numerical and (MRS2011B-2 (blue color) and MRS2011B-3 (red color)) semi-analytical solutions of the Moon rotation (for the DE406/LE406 ephemeris) 
Figure 9 explains why different semi-analytical solutions must be constructed for use on different time intervals.

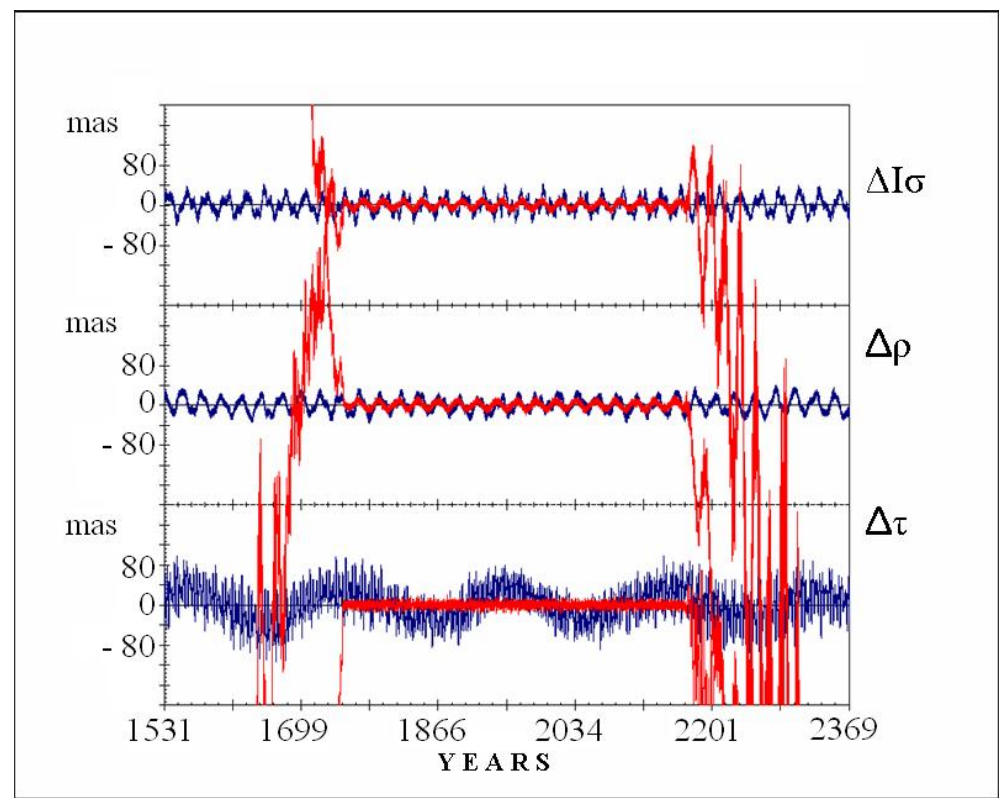

Fig. 9. Discrepancies between the numerical and (MRS2011B-1 (blue color) and MRS2011A-1 (red color)) semi-analytical solutions of the Moon rotation (for the DE406/LE406 ephemeris)

If the semi-analytical solution, which constructed for the application on a small time interval, continues to be used on longer time intervals, then the discrepancies between the numerical and MRS2011A-1 (curves of the red color in Figure 9) semi-analytical solutions of the Moon rotation are extremely large in comparison with the discrepancies between the numerical and MRS2011B-1 (curves of the blue color in Figure 9) semi-analytical solutions of the Moon rotation, where semi-analytical solution is constructed for the application on longer time intervals.

This investigation is finished at 6000 years time interval only for the DE406/LE406 ephemeris. In Figure 10 the discrepancies are depicted between the numerical and SMR semianalytical solutions of the Moon rotation over 6000 years.

The secular trend in the longitude of the descending node of epoch J2000 of the lunar equator longitude $\psi$ does not surpass 40 arc minutes over 6000 years. 


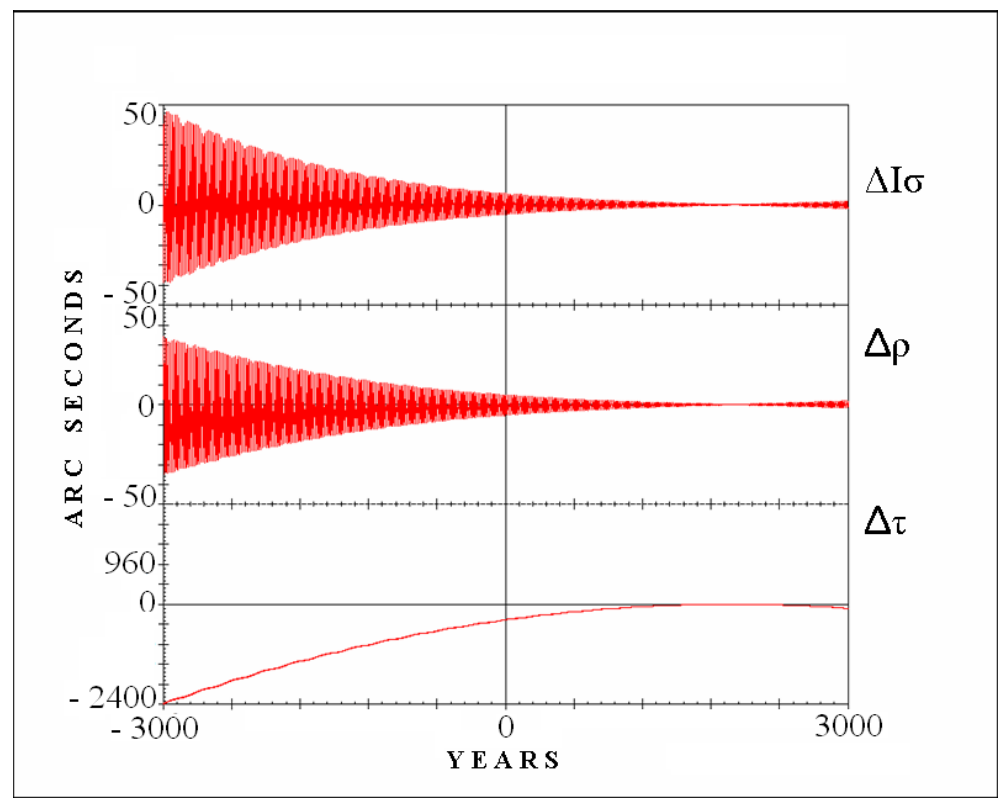

Fig. 10. Discrepancies between the numerical and SMR semi-analytical solutions of the

Moon rotation (for the DE406/LE406 ephemeris) over 6000 years

Figure 11 demonstrates the discrepancies between the numerical and MRS2011C-1 (blue color) semi-analytical solutions, after the first iteration, and the discrepancies between the numerical and MRS2011C-2 (red color) semi-analytical solutions, after the second iteration. The discrepancies in the libration angles decrease after the second iteration and are less than 8 arc seconds over 6000 years.

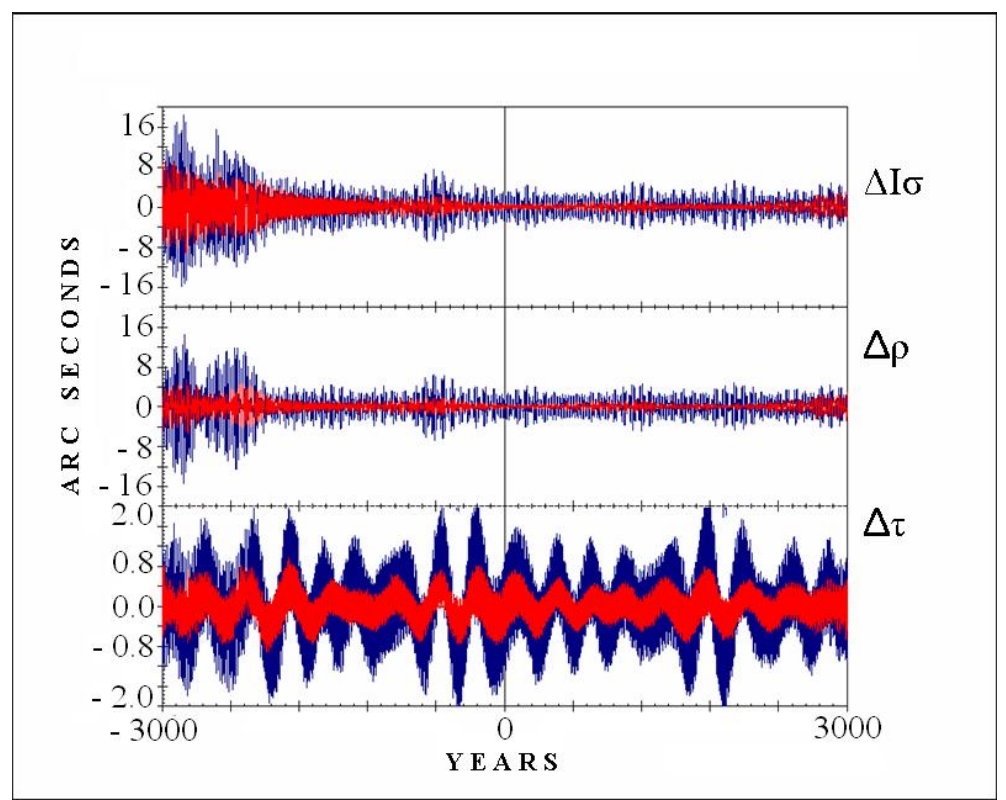

Fig. 11. Discrepancies between the numerical and (MRS2011C-1 (blue color) and MRS2011C-2 (red color)) semi-analytical solutions of the Moon rotation (for the DE406/LE406 ephemeris)

\section{CONCLUSIONS}

As the results of this investigation, the new high-precision Moon Rotation Series are constructed: 
MRS2011A, dynamically adequate to the DE404/LE404 (DE406/LE406) ephemeris, over 418 years,

MRS2011B, dynamically adequate to the DE404/LE404 (DE406/LE406) ephemeris, over 2000 years,

MRS2011C, dynamically adequate to the DE406/LE406 ephemeris, over 6000 years.

The periodic and Poisson parts of MRS2011A are very close to the periodic and Poisson parts of MRS2010, over all time interval of MRS2010, that evidences a good convergency of the iterative algorithm of this investigation.

The new more accurate series MRS2011 includes about 1523 secular, periodical and Poisson terms. The discrepancies between the numerical solution and MRS2011 do not surpass:

20 mas over 418 year time interval,

64 mas over 2000 year time interval,

8 arc seconds over 6000 year time interval.

It means a good consistency of MRS2011 series with the DE/LE ephemeris.

\section{ACKNOWLEDGEMENTS}

The authors are very grateful to Prof. Aleksander Brzezinski, Dr. Wieslaw Kosek for very useful discussions, Prof. Janusz Zielinski, Dr. Miloslawa Rutkowska, Dr. Leszek Jaworski and Dr. Ryszard Sdunek for the grants of financial support.

The investigation was carried out at the Central (Pulkovo) Astronomical Observatory of the Russian Academy of Science and the Space Research Centre of the Polish Academy of Science, under a financial support of the Cooperation between the Polish and Russian Academies of Sciences, Theme No 38 and the grants of Polish Academy of Science, No 5011-95 Dr. Ryszard Sdunek, 501-1-80 Prof. Janusz Zielinski, 123-033-49-17 PECS-98085 EEI Dr. Jaworski Leszek and Dr. Miloslawa Rutkowska.

\section{REFERENCES}

Belikov M.V. (1990): Methods of numerical integration with uniform and mean square approximation for solving problems of ephemeris astronomy and satellite geodesy, Manus. Geod., 15, No4, pp.182-200.

Eroshkin G.I., Taibatorov K.A., Trubitsina A.A. (1993): Constructing the specialized numerical ephemerides of the Moon and the Sun for solving the problems of the Earth's artificial satellite dynamics, ITA RAS, Preprint No 31, (in Russian).

Pashkevich V.V., Eroshkin G.I. (2010): Application of the spectral analysis for modeling the rotations of the Moon, Artificial Satellites, 45, 4, pp. 153-162.

Newhall X X and Williams J.G. (1997): Estimation of the lunar physical librations, Celestial Mechanics and Dynamical Astronomy, 66, pp. 21-30.

Received: 2011-12-08,

Reviewed: 2011-12-20, by Yu. Barkin,

Accepted: 2011-12-21. 